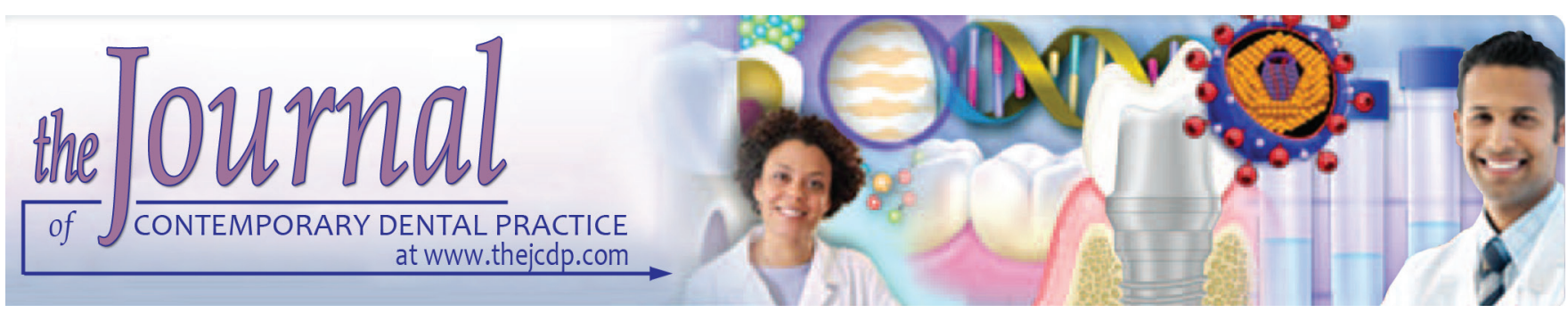

\title{
Spirometric Assessment of Impact of Complete Dentures on Respiratory Performance: An in vitro Study
}

\author{
${ }^{1} \mathrm{HS}$ Indrakumar, ${ }^{2}$ Deepa Venkatesh, ${ }^{3}$ Vidya V Adoni, ${ }^{4}$ Rucha Kashyap, ${ }^{5} \mathrm{D}$ Jayanthi, ${ }^{6}$ Nikhil Prakash
}

\begin{abstract}
Introduction: Edentulism is one of the debilitating states affecting a significant portion of the geriatric population. It is often considered as the ultimate marker for predicting the pressure and burden on oral health. Respiration is also a routine vital function running continuously through the human body and most commonly assessed by a spirometer. Hence, we planned the present study to assess the impact of complete dentures on the functioning of the respiratory system.
\end{abstract}

Materials and methods: In the present study, a spirometric assessment of the effect of complete dentures on respiratory performance was done. A total of 100 subjects were included, and diagnostic spirometer was used for carrying out the spirometric test at different stages of each subject. The spirometric test was carried out at four different stages: In the absence of both the denture (AODs), with both the dentures (maxillary and mandibular) inserted in the patient's mouth (BDs), with only maxillary denture inserted in the oral cavity (UDs), and finally, by inserting only the mandibular dentures in the oral cavity (LDs). Forced vital capacity (FVC), peak expiratory flow (PEF), forced expiratory volume in 1 second (FEV1), and forced expiratory flow between 25 and $75 \%\left(\mathrm{FEF}_{25-75}\right)$ were evaluated. All the results were compiled and assessed using Statistical Package for the Social Sciences (SPSS) software.

\footnotetext{
${ }^{1}$ Department of Prosthodontics, ESIC Dental College, Kalaburagi Karnataka, India

${ }^{2}$ Department of Dentistry, Kodagu Institute of Medical Sciences Madikeri, Karnataka, India

${ }^{3}$ Department of Prosthodontics, Mathrusri Ramabai Ambedkar Dental College and Hospital, Bengaluru, Karnataka, India

4,6 Department of Prosthodontics, Yogita Dental College and Hospital, Ratnagiri, Maharashtra, India

${ }^{5}$ Department of Periodontics, DR Mathrusri Ramabai Ambedkar Dental College and Hospital, Bengaluru, Karnataka, India

Corresponding Author: Deepa Venkatesh, Department of Dentistry, Kodagu Institute of Medical Sciences, Madikeri Karnataka India, Phone: +919880488466, e-mail: drdeepa. oralphysician@gmail.com
}

Results: Of the total 100 subjects included in the study, 42 were males and 58 were females. The mean FVC values of AOD, BD, LD, and UD group were 3.10, 3.02, 2.90, and 2.93 respectively. The mean PEF values of AOD, BD, LD, and UD group were $5.79,5.60,5.40$, and 5.48 respectively; $2.39,2.35,2.33$, and 2.32 were the mean FEV1 values observed in AOD, BD, LD, and UD group respectively. Statistically significant results were obtained while comparing AOD-FVC and BD-FVC and other oral conditions.

Conclusion: Spirometric values of respiratory functional tests in edentulous patients might be unfavorably affected by wearing complete dentures.

Clinical significance: Since subjects without complete dentures exhibited a maximum value of respiratory functional test, respiratory exercise protocols should also be carried in denturewearing edentulous patients for increasing the performance of the respiratory system.

Keywords: Complete denture, Edentulism, Spirometry.

How to cite this article: Indrakumar HS, Venkatesh D, Adoni VV, Kashyap R, Jayanthi D, Prakash N. Spirometric Assessment of Impact of Complete Dentures on Respiratory Performance: An in vitro Study. J Contemp Dent Pract 2018;19(2):177-180.

Source of support: Nil

Conflict of interest: None

\section{INTRODUCTION}

One of the debilitating states of the oral environment is edentulism. It is often considered as the ultimate marker for predicting the pressure and burden on oral health. Especially among geriatric patients, it remains a common problem affecting a significant population across the world. ${ }^{1,2}$ Various vital functions and processes are running continuously through the human body, and one of it is respiration. It involves the exchange of carbon dioxide and oxygen with the purpose of meeting the metabolic demands of the human body. 3,4

Spirometry is widely used for the assessment of the functioning of the respiratory system and its mechanical 
properties, by evaluating the lung volumes and capacities. Even in chronic pulmonary disorders, spirometry is routinely used. Spirometry offers numerous advantages in comparison with the other lung functional tests including its ability to evaluate through the noninvasive route. ${ }^{5-7}$ In the light of above-mentioned data, we planned the present study to assess the impact of complete dentures on the functioning of the respiratory system.

\section{MATERIALS AND METHODS}

The present study was conducted in the Department of Prosthodontics of the dental institute and included spirometric assessment of the effect of complete dentures on respiratory performance. Ethical approval was obtained from the Institutional Ethical Committee, and written consent was obtained from all the subjects after explaining in detail the entire research protocol.

A total of 100 subjects were included in the present study.

\section{Inclusion Criteria}

- Patients with complete edentulous dental arches

- Patients with a history of regular wearing of complete denture for a minimum time period of 5 years

- Complete denture-wearer patients, who were completely satisfied with their complete denture

- Patients with presence of Mallampati class I relationship of oral soft tissues

\section{Exclusion Criteria}

- Patients wearing complete denture that had fabrication faults

- Patients with a history of any systemic illness

- Patients with a history of any other pathology affecting primarily or secondarily the respiratory system, such as chronic obstructive pulmonary disease (COPD) or asthma

- Patients with a history of smoking

\section{Methodology Procedure}

Two hours before the start of the spirometric test, the patients were instructed not to perform any type of concentrated physical activity, including walking, running, or even climbing of the staircase. Patients were instructed to wear loose-fitting clothes so that unrestrained natural movement of the chest and abdomen could occur during the physiologic respiratory mechanism. Before the starting of the test, the height and the weight of all the subjects were noted. Experienced technicians were hired for performing the spirometry procedure. The diagnostic spirometer (Technocare Medisystems, India) was used for carrying out the spirometric test at different stages of each subject. Spirometric test was carried out at following stages:

- Intimal testing was done in subjects with the absence of dentures in physiologic oral conditions AODs. The values obtained thus were regarded as the control values

- Second testing was done with both the dentures (maxillary and mandibular) inserted in the patient's mouth and was designated as BDs

- Third testing was done in subjects by inserting only maxillary denture in the oral cavity, and the values were designated as UDs

- Final testing was done in subjects by inserting only the mandibular dentures in the oral cavity, and the values obtained were designated as LDs.

Measurement of the following spirometric parameters was done in all the patients:

- FVC

- PEF, FEV1 and

- $\mathrm{FEF}_{25-75}$.

All the results were compiled and noted on the Excel sheet. Comparison in between the values obtained at different stages was done using SPSS software. Chi-square test, Fisher t-test, and one-way analysis of variance were used for assessment of the level of significance; $p<0.05$ was considered as statistically significant.

\section{RESULTS}

A total of 100 subjects were included in the present study. Of the 100, 42 were males and 58 were females. Mean age of all the subjects was 52.4 years, while the mean body mass index was $29.5 \mathrm{~kg} / \mathrm{m}^{2}$ (Graph 1). Mean spirometric values of various oral health status conditions are summarized in Table 1. The mean FVC values of AOD, BD, LD, and UD group were 3.10, 3.02, 2.90, and 2.93 respectively. The mean PEF values of AOD, BD, LD, and UD group were $5.79,5.60,5.40$, and 5.48 respectively; 2.39 , 2.35, 2.33,

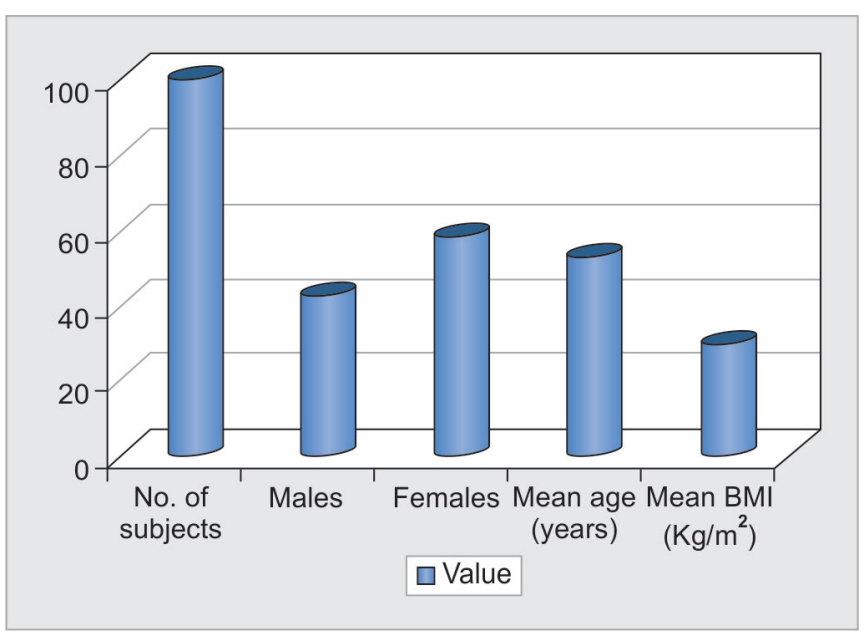

Graph 1: Demographic details of all the subjects 
Spirometric Assessment of Impact of Complete Dentures on Respiratory Performance

Table 1: Mean value of spirometric parameters of all the subjects in various oral conditions

\begin{tabular}{ll}
\hline Oral health status & Mean value \\
\hline AODs-FVC & 3.10 \\
BDs-FVC & 3.02 \\
LDs-FVC & 2.90 \\
UDs-FVC & 2.93 \\
AODs-PEF & 5.79 \\
BDs-PEF & 5.60 \\
LDs-PEF & 5.40 \\
UDs-PEF & 5.48 \\
AODs-FEV1 & 2.39 \\
BDs-FEV1 & 2.35 \\
LDs-FEV1 & 2.33 \\
UDs-FEV1 & 2.32 \\
AODs-FEF $25-75$ & 2.79 \\
BDs-FEF & $25-75$ \\
LDs-FEF $_{25-75}$ & 2.62 \\
UDs-FEF $25-75$ & 2.68 \\
\hline
\end{tabular}

and 2.32 were the mean FEV1 values observed in AOD, $\mathrm{BD}, \mathrm{LD}$, and $\mathrm{UD}$ group respectively. The $\mathrm{FEF}_{25-75}$ values for AOD, BD, LD, and UD group were found to be 2.79, $2.62,2.68$, and 2.67 respectively. Statistically significant results were obtained while comparing the following cases as shown in Table 2:

- AODs-FVC and BDs-FVC

- AODs-FVC and LDs-FVC

- AODs-FVC and UDs-FVC

- AODs-PEF and LDs-PEF

- AODs-PEF and UDs-PEF

- AODs-FEV1 and BDs-FEV1

- AODs-FEV1 and LDs-FEV1

- AODs-FEV1 and UDs-FEV1

- AODs-FEF $25-75$ and BDs-FEF $25-75$, and

- AODs-FEF $25-75$ and UDs-FEF $25-75$.

\section{DISCUSSION}

Ever since the evolution of modern dentistry, dentists have had a keen interest in the effect of skeletal soft tissue changes on the facial soft-tissue profile of an individual. Correlation between various cephalometric variables and morphology of face along with the relationship of cervical and orofacial areas have been a topic of current research for the past few decades. ${ }^{8-10}$ Hence, we planned the present study to assess the impact of complete dentures on the functioning of the respiratory system.

In the present study, we analyzed a total of 100 subjects and evaluated the effect of various oral conditions [with and without dentures (WODs)] on the respiratory performance (Graph 1). Spirometric values were highest in the edentulous condition in the absence of denture (Table 1). Furthermore, we observed significant effect of denture wearing on spirometric values in edentulous patients ( $\mathrm{p}<0.05$; Table 2). As demonstrated by various past
Table 2: Comparative evaluation of spirometric values with and without dentures

\begin{tabular}{ll}
\hline Comparative evaluation of spirometric values & $p$-value \\
\hline AODs-FVC and BDs-FVC & $0.02^{*}$ \\
AODs-FVC and LDs-FVC & $0.03^{*}$ \\
AODs-FVC and UDs-FVC & $0.01^{*}$ \\
AODs-PEF and BDs-PEF & 0.82 \\
AODs-PEF and LDs-PEF & $0.01^{*}$ \\
AODs-PEF and UDs-PEF & $0.02^{*}$ \\
AODs-FEV1 and BDs-FEV1 & $0.04^{*}$ \\
AODs-FEV1 and LDs-FEV1 & $0.04^{*}$ \\
AODs-FEV1 and UDs-FEV1 & $0.02^{*}$ \\
AODs-FEF & $0.01^{*}-75$ and BDs-FEF \\
AODs-75 $_{25 E F}$ & 0.06 \\
AODs-75 $_{25 E F}$ and LDs-75 and UDs-FEF & \\
\hline
\end{tabular}

*Statistically significant

studies, a strict relationship exists between orofacial conditions and the upper airway. ${ }^{11,12}$ Oral cavity is narrowed by wearing of large prosthesis like complete dentures. Further, posterior positioning of the tongue occurs by wearing of complete denture due to its high thickness, which further results in collapsing of pharyngeal airspace. ${ }^{8}$ It was intended by Carossa et $\mathrm{al}^{13}$ to evaluate whether edentulism may affect spirometric measurements. In a total of 58 edentulous subjects, they recorded spirometric values in with denture and WOD conditions. They also assessed 36 asymptomatic normal subjects $(\mathrm{N})$ and 22 patients with COPD. They used the cephalometry technique for assessment of the retropharyngeal space with denture and WOD in 10 subjects. Removing denture in the $\mathrm{N}$ group created a noteworthy decline in lung volumes and airflow rates, whereas it was unsuccessful in COPD patients. Furthermore, it showed retropharyngeal space, which was meaningfully reduced by removing dentures in both the groups. It was also observed that by decreasing extrathoracic airway caliber, edentulism influences significantly the spirometric measurements in normal subjects, but not in those with COPD.

In another study, immediate daily and weekly variations in respiratory resistance (Rrs) measured by means of the forced oscillation technique (FOT) to spirometric indices were compared in pediatric patients with chronic respiratory symptoms. They made investigations in relation to lung functioning measurements on 19 children in 4 days, i.e., two consecutive days during two consecutive weeks. During the entire study period, the measurements were conducted at the same time of the day and every time repeating three times. Further, in 12 children, they observed that intrasubject factors of deviation (coefficient of variation, $\mathrm{CoVs}$ ) for Rrs and FOT were more than those for spirometric indices. However, in the instantly repetitive measurements, the $\mathrm{CoV}$ was of maximal expiratory flow at $25 \%$ vital capacity larger than that of Rrs, FOT. ${ }^{14}$ 
Bucca et $\mathrm{al}^{15}$ noted the alterations in values of FVC, FEV1, PEF rate, FEF (50\%), FIV1, and FIF (50\%) recorded with denture and WOD in three gatherings of edentulous subjects: 36 asymptomatic subjects with typical spirometry (N), 22 patients with unending obstructive aspiratory illness (COPD), and 18 with interstitial lung disease (ILD). They used cephalometry for evaluation of retropharyngeal space in patients with denture and WOD. Subjects with $\mathrm{N}$ and ILD had fundamentally had air movement rates brought down WOD, while subjects with COPD had no noteworthy contrast in spirometric values recorded with denture or WOD. The retropharyngeal space was fundamentally diminished by eradicating dentures. These conclusions demonstrate that in edentulous subjects with a usual or preventive configuration, the account of stream volume arcs with denture or WOD delivers little, yet noteworthy alterations. Although such contrasts do not seem to have clinical essentialness, the point that when dentures are utilized, some respiratory streams are higher would support the utilization of dentures in edentulous subjects throughout spirometric assessment.

In 46 edentulous subjects, in another study, spirometric performance was analyzed. Respiratory utilities of the subjects were assessed by spirometric tests that were performed in four diverse oral conditions: WOD, with dentures, bring down denture just, and upper denture as it were. The FVC, top expiratory stream, constrained expiratory volume in 1 second, and constrained expiratory stream in the vicinity of 25 and $75 \%$ were assessed. Important variations were found between spirometric parameters in various oral conditions. In all spirometric parameters, the most imperative noteworthy variation was found between conditions WOD, FVC, and with lower dentures FVC and WOD and with upper dentures. It was noticed that entire dentures may adversely influence spirometric estimations of edentulous subjects. Nonetheless, existing conclusions should be affirmed with progressive respiratory utility. ${ }^{16}$

\section{CONCLUSION}

The FCV, PEF, FEV1, and FEF $_{25-75}$ spirometric parameters are affected by wearing of complete dentures in edentulous patients. Since there are numerous respiratory functional tests available these days with added advantages, testing of these results with more advanced tests is required for the establishment of above-obtained data.

\section{Clinical Significance}

Dentures may adversely influence spirometric estimations of edentulous subjects. Protocols for respiratory exercise should be carried in denture-wearing edentulous patients for increasing the performance of the respiratory system.

\section{REFERENCES}

1. Müller F, Naharro M, Carlsson GE. What are the prevalence and incidence of tooth loss in the adult and elderly population in Europe? Clin Oral Implants Res 2007 Jun;18 (Suppl3):2-14.

2. Beltrán-Aguilar ED, Barker LK, Canto MT, Dye BA, Gooch BF, Griffin SO, Hyman J, Jaramillo F, Kingman A, NowjackRaymer R, et al. Surveillance for dental caries, dental sealants, tooth retention, edentulism, and enamel fluorosis-United States, 1988-1994 and 1999-2002. MMWR Surveill Summ 2005 Aug;54(3):1-43.

3. Douglass CW, Shih A, Ostry L. Will there be a need for complete dentures in the United States in 2020? J Prosthet Dent 2002 Jan;87(1):5-8.

4. Millar WJ, Locker D. Edentulism and denture use. Health Rep 2005 Nov;17(1):55-58.

5. Buist AS, McBurnie MA, Vollmer WM, Gillespie S, Burney P, Mannino DM, Menezes AM, Sullivan SD, Lee TA, Weiss KB, et al. International variation in the prevalence of COPD (the BOLD study): a population-based prevalence study. Lancet 2007 Sep;370(9589):741-750.

6. Menezes AM, Perez-Padilla R, Jardim JR, Muiño A, Lopez MV, Valdivia G, Montes de Oca M, Talamo C, Hallal PC, Victora CG, et al. Chronic obstructive pulmonary disease in five Latin American cities (the PLATINO study): a prevalence study. Lancet 2005 Nov;366(9500):1875-1881.

7. Salvi SS, Barnes PJ. Chronic obstructive pulmonary disease in non-smokers. Lancet 2009 Aug;374(9691):733-743.

8. Gokce HS, Gokce S, Akin E, Bengi O. Effects of complete denture wearing on the head posture and posterior airway space. J Dent Sci 2011 Mar;6(1):6-13.

9. Haikola B, Oikarinen K, Söderholm AL, Remes-Lyly T, Sipilä K. Prevalence of edentulousness and related factors among elderly finns. J Oral Rehabil 2008 Nov;35(11):827-835.

10. Turner M, Jahangiri L, Ship JA. Hyposalivation, xerostomia and the complete denture: a systematic review. J Am Dent Assoc 2008 Feb;139(2):146-150.

11. Choi JK, Hur YK, Lee JM, Clark GT. Effects of mandibular advancement on upper airway dimension and collapsibility in patients with obstructive sleep apnea using dynamic upper airway imaging during sleep. Oral Surg Oral Med Oral Pathol Oral Radiol Endod 2010 May;109(5):712-719.

12. Lowe AA, Gionhaku N, Takeuchi K, Fleetham JA. Threedimensional CT reconstructions of tongue and airway in adult subjects with obstructive sleep apnea. Am J Orthod Dentofacial Orthop 1986 Nov;90(5):364-374.

13. Carossa S, Pera P, De LilloA, Corsalini M, LombardoS, Bucca C. The influence of edentulism on spirometric values. Minerva Stomatol 2000 Sep;49(9):405-408.

14. Timonen KL, Randell JT, Salonen RO, Pekkanen J. Short-term variations in oscillatory and spirometric lung function indices among school children. Eur Respir J 1997 Jan;10(1):82-87.

15. Bucca CB, Carossa S, Colagrande P, Brussino L, Chiavassa G, Pera P, Rolla G, Preti G. Effect of edentulism on spirometric tests. Am J Respir Crit Care Med 2001 Mar;163(4):1018-1020.

16. Piskin B, Sipahi C, Karakoc O, Atay A, Ciftci F, Tasci C, Akin H, Arisan V, Sevketbeyoglu H, Turker T. Effects of complete dentures on respiratory performance: spirometric evaluation. Gerodontology 2014 Mar;31(1):19-24. 